\title{
Detection of a heterozygous germline APC mutation in a three-generation family with familial adenomatous polyposis using targeted massive parallel sequencing in Vietnam
}

Hoa Giang ${ }^{2,7^{*+}}$, Vu T Nguyen ${ }^{6+}$, Sinh D Nguyen ${ }^{9}$, Huu-Phuc Nguyen ${ }^{3,4}$, Binh T Vo ${ }^{1,8}$, Truc M Nguyen ${ }^{1,8}$, Nguyen H Nguyen ${ }^{2,5}$, Kiet D Truong ${ }^{7}$, Thanh-Thuy T Do ${ }^{1}$, Minh-Duy Phan ${ }^{2^{*}}$ and Hoai-Nghia Nguyen ${ }^{1 *} \mathbb{D}$

\begin{abstract}
Background: Familial adenomatous polyposis (FAP) is an autosomal dominant hereditary syndrome characterised by the development of hundreds to thousands of adenomatous colonic polyps during the second decade of life. FAP is caused by germ line mutations in the adenomatous polyposis coli (APC) gene located on chromosome $5 \mathrm{q} 21-22$.

Case presentation: A 36-year-old female was presented with 100-1000 adenomatous colonic polyps, typical of classic FAP symptoms. Genetic testing using massively parallel sequencing identified a 5-bp deletion (c.3927_3931delAAAGA) which causes frameshift (p.Glu1309Aspfs) and creates a premature stop codon, resulting in the replacement of the last 1535 amino acids of APC by five incorrect amino acids. Two of the proband's four siblings also exhibited classic FAP symptoms and carried the same 5-bp heterozygous deletion in the APC gene. One of the proband's two nephews also tested positive for this mutation but has not been examined by endoscopy due to his young age.

Conclusions: We reported here for the first time the use of massively parallel sequencing (MPS)-based genetic testing to identify a germline mutation within a three-generation Vietnamese family. This mutation is most likely responsible for the development of FAP.
\end{abstract}

Keywords: Familial adenomatous polyposis, APC gene, Germline mutation, Genetic testing, Massively parallel sequencing

\section{Background}

Familial adenomatous polyposis (FAP) is an autosomal dominant hereditary syndrome characterised by the development of hundreds to thousands of adenomatous colonic polyps during the second decade of life. FAP has an estimated prevalence of once in every 10,000 births [1] and if left untreated, colon cancer is essentially inevitable. The mean age of colon cancer diagnosis is 39 years

\footnotetext{
* Correspondence: gianghoa@gmail.com; pmduy@yahoo.com; nhnghia81@gmail.com

${ }^{\dagger}$ Hoa Giang and Vu T Nguyen contributed equally to this work.

${ }^{2}$ Gene Solutions, Ho Chi Minh city, Vietnam

${ }^{1}$ Center for Molecular Medicine, University of Medicine and Pharmacy, Ho

Chi Minh city, Vietnam

Full list of author information is available at the end of the article
}

and $7 \%$ of untreated individuals develop cancer by age 21 and $90 \%$ by age 45 [2]. FAP is caused by germ line mutations in the adenomatous polyposis coli $(A P C)$ gene located on chromosome 5q21-22 [1]. Apart from FAP, mutations in $A P C$ are also associated with attenuated FAP, and gastric adenocarcinoma and proximal polyposis of the stomach (GAPPS). Currently there are more than 750 mutations in the $A P C$ gene that were classified as pathogenic or likely pathogenic in ClinVar database. The majority of disease-causing mutations identified to date are gene inactivating and result in APC protein truncation [1]. It is known that patients with identical mutations can develop different clinical features [3]. However, some correlations between the location of mutations in 
the $A P C$ gene and phenotype have been observed. For example, the average age of onset in individuals with colonic symptoms varied by mutation locations, with onset age of 20 years for mutations at codon 1309, age of 30 years for mutations between 168 and 1580 and age of 52 years for mutations at $5^{\prime}$ of codon 168 and $3^{\prime}$ of codon 1580 [4].

Individuals who have clinical diagnosis or clinical suspicion of FAP or who are at-risk family members or who have as few as 10-15 adenomatous polyps might benefit from genetic testings [5]. In recent years, genetic tests using massively parallel sequencing (MPS) technologies have become more affordable. However, the adoption rates of such MPS-based genetic tests in developing countries such as Vietnam remain very low. In this study, we report the use of MPS to identify a mutation in the APC gene that are linked with FAP in a family of three generations in Vietnam.

\section{Case presentation}

\section{Clinical information}

A 36-year-old female (proband, II-2, Fig. 1) had reported to the Department of Oncology, Thu Duc hospital (Ho Chi Minh City, Vietnam) with recurrence of frequent diarrhea and stool mixed with blood and mucus. Endoscopy revealed 100-1000 colonic polyps with the size in range of 5-15 mm (Fig. 2). Colonic polypectomy was later performed to prevent the development of colonic cancer. Family history investigation revealed that the proband's father died of colorectal cancer at the age of 51 year. Endoscopic screenings were therefore performed on all of her siblings. Two of her four siblings (II-5 and II-6) also exhibited 100-1000 colonic polyps suggesting of FAP syndrome in this family (Fig. 2). These patients did not exhibited other non-colonic manifestations such as congenital hypertrophy of the retinal pigment epithelium (CHRPE) or desmoid tumors.

\section{Mutation analysis}

To identify the genetic mutation(s) that might have caused the FAP in this family, a MPS-based oncoSure hereditary cancer test (Gene Solutions, Vietnam) was chosen to perform on all nine members of the family including the proband, her spouse, all of her siblings and their spouses as well as their offsprings. The oncoSure hereditary cancer test is a 17-gene panel including BRCA1, BRCA2, PALB2, PTEN, TP53, CDH1, MLH1, MSH2, MSH6, PMS2, EPCAM, APC, MUTYH, STK11,VHL, RB1, RET that identifies an elevated risk for 10 hereditary cancers: breast, ovarian, colorectal, endometrial, gastric, pancreatic,

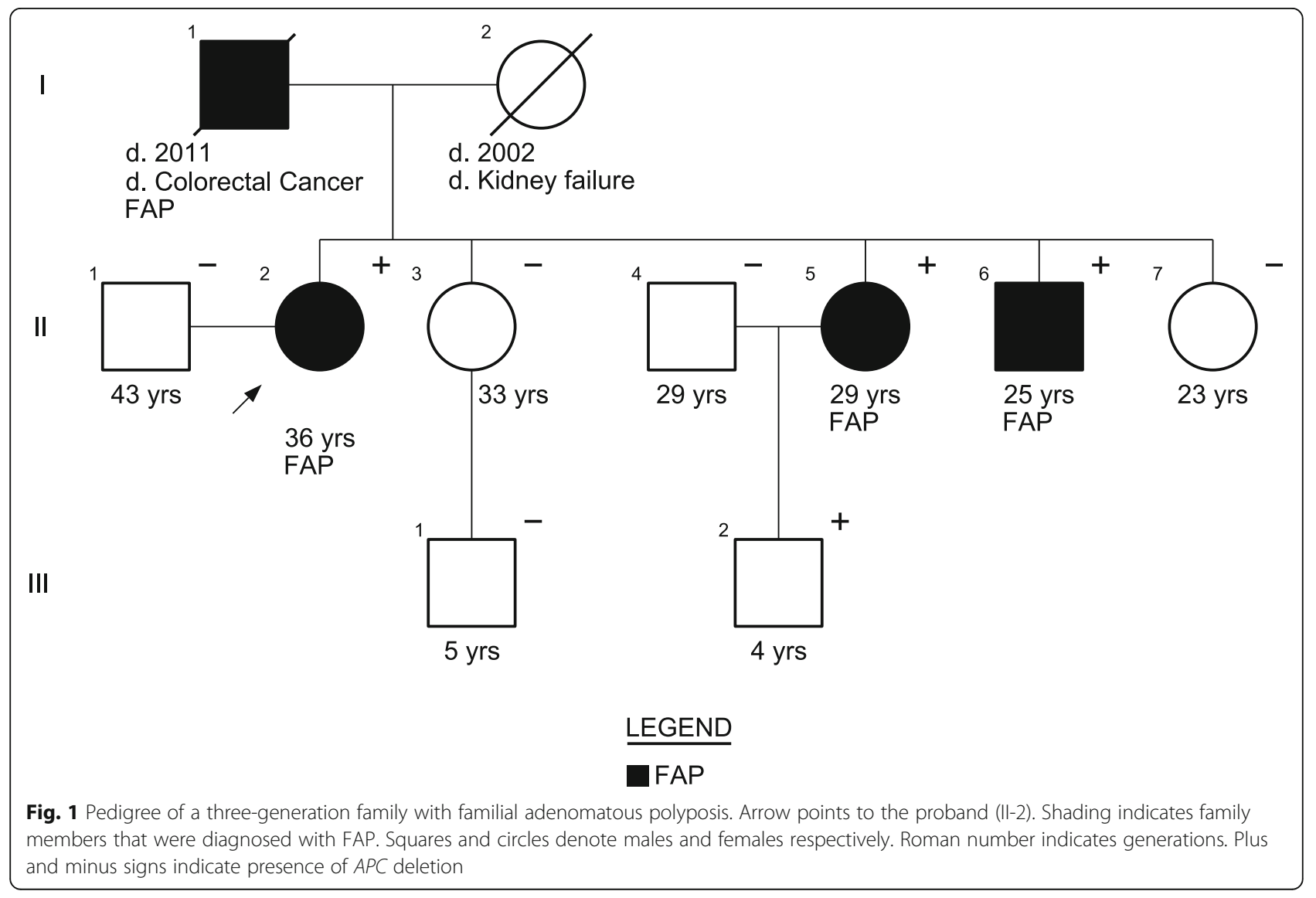




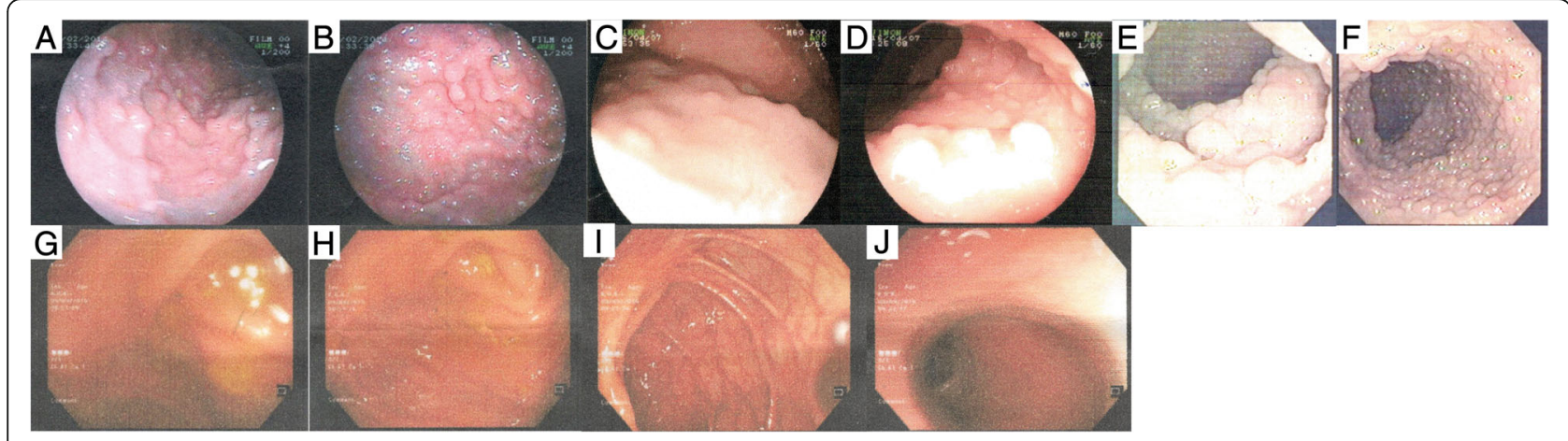

Fig. 2 Colonoscopy analysis of affected and unaffected members. a-f Familial adenomatous polyp: multiple polyps in the colon of affected members (A-B: II-2, C-D: II-5, E-F: II-6, respectively). g-j Polyps were not observed in unaffected members (G-H: I--3, I-J: II7)

prostate, melanoma, endocrine and retinoblastoma. Blood samples were collected and genomic DNA were extracted with QiaAmp DNA blood mini kit from Qiagen (Hilden, Germany) following the manufacturer's instructions. DNA fragmentation and library preparation were done using NEBNext Ultra II DNA Library Prep Kit from New England BioLabs (Ipswich, MA, USA). Pool of sequencing libraries was captured using predesigned probes for 17 target genes from IDTDNA (Coralville, IA, USA). The targeted sequences covered all exons and a small flanking sequence of introns. Captured products were amplified with KAPA HiFi HotStart ReadyMix from KAPA Biosystems (Wilmington, MA, USA). Samples were sequenced on Illumina MiniSeq platform (Illumina, San Diego, CA, USA). Raw sequences from each sample were aligned to the reference human genome from University of California, Santa Cruz (UCSC) Genome Browser (NCBI build GRCh38) using Burrows Wheeler Aligner (BWA) [6]. The aligned output was used to compute depth and breadth of coverage in the target region, and SNP/INDEL calling with GATK standard pipeline [7]. Variants were classified using ClinVar database (National Institutes of Health).

A heterozygous deletion c.3927_3931delAAAGA (p.Glu1309Aspfs) in the APC gene was found in the proband (II-2), all other affected members (II-5 and II-6) and a young male member in the third generation (4 years of age, III-2). No other pathogenic or likely pathogenic mutations was detected elsewhere in the genes targeted by the oncoSure hereditary cancer gene panel. This deletion was absent in all unaffected members (II-3 and II-7) and a 5 year-old third generation male member (III-1) (Table 1). We did not identify this mutation in the 50 normal control of the same ethnic origin and age range.

This 5-bp deletion occurs at codon 1309 which causes frameshift and creates a premature stop codon at position 4 of the new reading frame, resulting in the replacement of the last 1535 amino acids of APC by three incorrect amino acids. This variant was predicted to cause loss of normal protein function through protein truncation. The truncated protein caused by this deletion is similar to that caused by another 5-bp deletion variant (NM_000038.5(APC):c.3920_3924delTAAAA) which was reported in two individuals for whom clinical APC testing was ordered (Evidence detailed provided by GeneDx in ClinVar).

\section{Confirmation of the novel mutation by sanger sequencing}

To confirm this novel heterozygous deletion, we performed PCR and Sanger sequencing for all tested cases.

Table 1 Clinical and genetic characteristics of the affected and unaffected family members

\begin{tabular}{|c|c|c|c|c|c|}
\hline ID & Sex & Present Age & Age at diagnosis & Colonscopy screening & APC mutation (c.3927_3931delAAAGA) \\
\hline$\|-1$ & Male & 43 & NA & NA & No \\
\hline$\|-2$ & Female & 36 & 24 & 100-1000 colonic polyps (5-15 mm) & Yes \\
\hline $11-3$ & Female & 33 & 24 & $<10$ colonic polyps & No \\
\hline$\|-4$ & Male & 29 & NA & NA & No \\
\hline$\|-5$ & Female & 29 & 20 & 100-1000 colonic polyps (5-15 mm) & Yes \\
\hline$\| 1-6$ & Male & 25 & 18 & 100-1000 colonic polyps (5-15 mm) & Yes \\
\hline$\|-7$ & Female & 23 & 13 & $<10$ colonic polyps & No \\
\hline|| $\mid-1$ & Male & 5 & NA & NA & No \\
\hline|| $\mid-2$ & Male & 4 & NA & NA & Yes \\
\hline
\end{tabular}




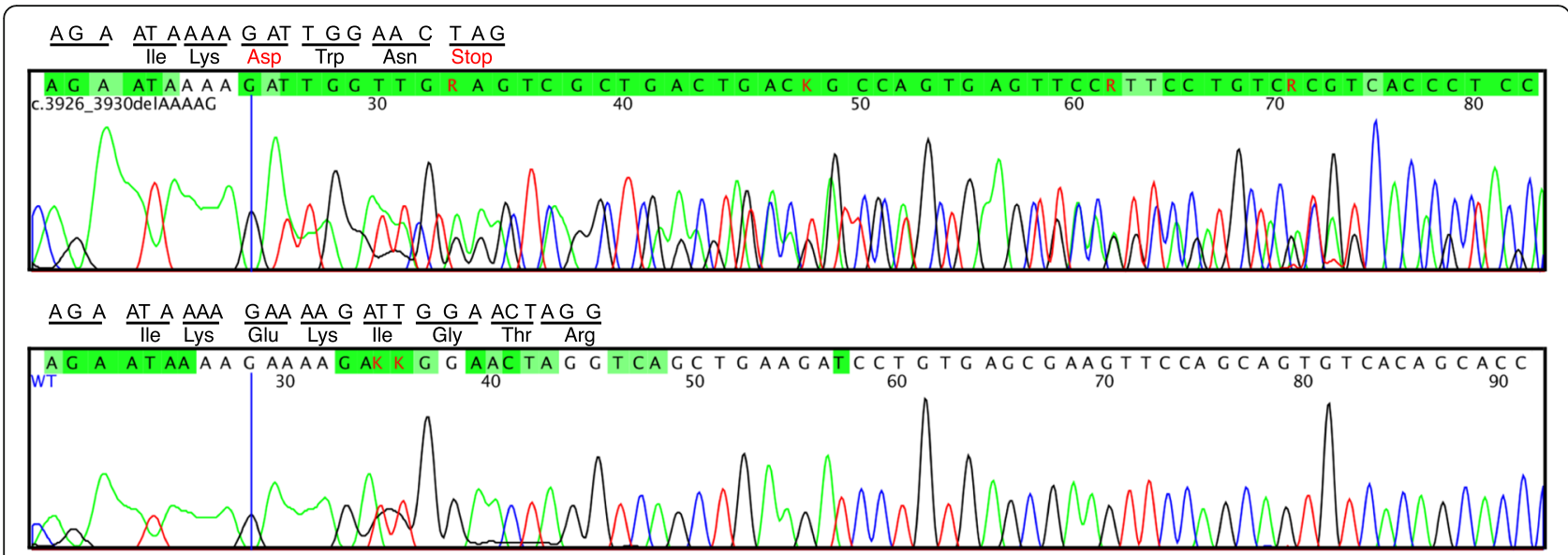

Fig. 3 Validation of the deletion in affected members using Sanger sequencing. (Top) The heterozygous deletion c.3927_3931delAAAGA in proband II-2: the deletion causes a frameshift and creates a Stop codon downstream. (Bottom) The complete sequence in unaffected members. The blue vertical line represents the last base before deletion

We designed primers flanking the deletion location using the reference human genome from UCSC Genome Browser. The primers (5'ATCAGACGACACAG GAAGCA-3' and 5' - ACTCAGGCTGGATGAACAA GA-3') were synthesized and purified by IDTDNA (Coralville, IA, USA). PCR amplification was prepared using Q5 High-Fidelity $2 \times$ Master Mix from New England BioLabs (Ipswich, MA, USA) following manufacturer's instructions. The PCR products were sequenced using Applied Biosystems 3500xl. As shown in Fig. 3, the 5 -nucleotide deletion was found in the proband but not in the unaffected member, confirming our finding using MPS technology.

\section{Discussion and conclusions}

Familial adenomatous polyposis (FAP) is characterised by the development of hundreds to thousands of adenomatous colonic polyps during the second decade of life, and is a disease predisposing individuals to colorectal cancer (CRC)
[1]. In this pedigree, the proband presented with rectal bleeding in combination with change in bowel habits, which was the most common symptom combination from the local tumour of CRC $[8,9]$, and endoscopic diagnosis showed symptoms of classic FAP (100-1000 polyps). A 5-bp heterozygous deletion c.3927_3931delAAAGA was detected in the $A P C$ gene, resulting in frameshift mutation (p.Glu1309Aspfs). The heterozygous mutation was suggestive of inheritance of this mutation from her father who had died of CRC. Unfortunately, we were unable to confirm the presence of this mutation in the proband's father. Consistent with the theory that the proband inherited a wild-type $A P C$ allele from her mother and the mutant $A P C$ allele from her father, two of her four siblings (II-5 and II-6) also exhibited classic FAP symptoms and carried the same 5-bp heterozygous deletion in the APC gene. The proband's nephew (III-2), son of her sister (II-5), also carried this heterozygous mutation but had not been examined by endoscopy due to his young age (4 years old). It was therefore

Table 2 Reports of c.3927_3931delAAAGA mutation associated with FAP and/or colorectal tumour

\begin{tabular}{lllll}
\hline Study & Year & Mutation type & Detection technology & Country \\
\hline Miyoshi et al. [14] & 1992 & Germline & PCR-Sanger sequencing & Japan \\
Cottrell et al. [15] & 1992 & Germline + Somatic & PCR-SSCP-Sanger sequencing & UK \\
Varesco et al. [16] & 1993 & Germline & PCR-SSCP-Sanger sequencing & PCR-SSCP-Sanger sequencing \\
De Benedetti et al. [17] & 1994 & Somatic & PCR-SSCP-Sanger sequencing & Italy \\
Yagi et al. [18] & 1997 & Somatic & PCR-Sanger sequencing & Japan \\
Gryfe et al. [19] & 1998 & Somatic & PCR-Sanger sequencing & Canada \\
Sánchez-de-Abajo et al. [20] & 2007 & Somatic & PCR-SSCP/ PCR-Sanger sequencing & India \\
Syed Sameer et al. [21] & 2011 & Somatic & PCR-Sanger sequencing & Australia \\
Christie et al. [22] & 2013 & Somatic & MPS & China \\
Chen et al. [23] & 2015 & Germline & &
\end{tabular}


crucial for the carriers in this family to have routine medical monitoring to properly manage and prevent the development of CRC. Any new offsprings from the carriers should also undertake genetic testings to determine the status of their $A P C$ alleles in order to have proper genetic counselling and early medical intervention.

FAP is caused by germ line mutations in the tumor suppressor gene, Adenomatous Polyposis Coli (APC), located on chromosome 5q21-q22 [1]. At the time of writing this report (Jun 2018), the ClinVar database [10] recorded 4471 variants in the APC gene, 609 of which were Pathogenic variants and 175 were Likely pathogenic. This c.3927_3931delAAAGA variant causes a frameshift (p.Glu1309Aspfs), results in a truncated version of $A P C$ gene. It falls between codons 169 to 1393, which are generally associated with classic FAP $[3,11]$ or more specifically, it falls in a region from codon 1250 to 1464 that is associated with severe FAP [12]. Furthermore, the truncation at codon 1309 has been shown to exert a dominant negative effect of $A P C$ gene products by inhibition of the wild-type APC activity [13]. The $100 \%$ correlation between the carriage of this mutation and FAP symptoms in all members over 20 years of age in our study further strengthens the interpretation of this variant as pathogenic. This mutation was first reported by Miyoshi et al. in a study searching for germline mutations of the $A P C$ gene in 79 unrelated FAP patients from the US and Japan [14]. This mutation was later reported as germline and somatic mutation in several other countries (Table 2). To the best of our knowledge, this is the first report using MPS to identify a variant in the $A P C$ gene in a Vietnamese pedigree.

The clinical application of MPS in the field of oncology has gained significant development and adoption in recent years. Mutation detection in hereditary cancer syndromes has major impact in genetic counselling, helps increase the chance of survival, defines the prognosis of carriers and identifies the most appropriate and personalised prophylactic measures [24]. In this study, we used a 17-gene panel (oncoSure) to detect germline mutations associated with 10 different hereditary cancer syndromes including Lynch syndrome, FAP and MUTY$H$-associated polyposis. Using this panel, we have successfully identified a mutation in the APC gene associated with FAP in a three-generation family. This mutation was verified by Sanger sequencing on all affected family members, validating our targeted MPS tests. MPS-based oncology research and clinical genetic testings are still relatively new in Vietnam and thus there is little information about the variants specific to Vietnamese population. This study is the first effort to report cases of cancer-associated germline variants found in the Vietnamese population. However, continuous surveillance using MPS technology is needed to build a comprehensive catalogue of pathogenic variants specific to Vietnam.

In conclusion, we reported a heterozygous mutation in the $A P C$ gene that was found in multiple members of a three-generation family in Vietnam. This mutation is most likely responsible for development of the FAP symptoms in carriers. We have deposited the information of this novel mutation to ClinVar (Variation ID: 816). To our knowledge, this is the first study in Vietnam that used MPS for the identification of cancer linked mutations.

\section{Abbreviations \\ APC: Adenomatous polyposis coli; CRC: Colorectal cancer; FAP: Familial adenomatous polyposis; MPS: Massive parallel sequencing; PCR: Polymerase chain reaction; SSCP: Single-strand conformation polymorphism}

\section{Acknowledgements}

We thank all the subjects for their participation.

Funding

This work was supported by Gene Solutions (GS002).

Availability of data and materials

The datasets used and analysed during the current study available from the corresponding author on reasonable request.

\section{Authors' contributions}

VTN, SDN, PHN, NHN collected the samples and did the follow-up. BTV, TMN, MDP, HG performed experiments and did the analysis. MDP, TTD, KDT wrote the manuscript. HG, MDP, HNN designed the study, supervised the study and revised the manuscript. All of the co-authors have read this manuscript and support this submission. All authors read and approved the final manuscript.

\section{Ethics approval and consent to participate}

This study was approved by the Ethical Committee of the University of Medicine and Pharmacy at Ho Chi Minh city according to the Helsinki Principles. All adult participants have given written consent to participate in this study. The proband and affected family members have been diagnosed by oncologists and pathologists according to clinical guidelines from American College of Gastroenterology (ACG).

\section{Consent for publication}

Written informed consents for publication of their clinical details and images were obtained from all adult patients. Consents were given by the parents on behalf of the minors (those under the age of 18) included in this study.

\section{Competing interests}

HG, NHN and MDP are employed by Gene Solutions, the company providing genetic testing using oncoSure gene panel.

\section{Publisher's Note}

Springer Nature remains neutral with regard to jurisdictional claims in published maps and institutional affiliations.

\section{Author details}

${ }^{1}$ Center for Molecular Medicine, University of Medicine and Pharmacy, Ho Chi Minh city, Vietnam. ${ }^{2}$ Gene Solutions, Ho Chi Minh city, Vietnam.

${ }^{3}$ Department of Oncology, University of Medicine and Pharmacy, Ho Chi Minh city, Vietnam. ${ }^{4}$ Ung Buou Hospital, Ho Chi Minh city, Vietnam. ${ }^{5} \mathrm{Gia}$ Dinh Hospital, Ho Chi Minh city, Vietnam. ${ }^{6}$ Thu Duc Hospital, Ho Chi Minh city, Vietnam. ${ }^{7}$ Medical Genetics Institute, Ho Chi Minh city, Vietnam.

${ }^{8}$ Graduate Program of Genetics, University of Science, Ho Chi Minh city, Vietnam. ${ }^{9}$ Vinmec Central Park International Hospital, Ho Chi Minh city, Vietnam. 
Received: 29 June 2018 Accepted: 1 October 2018

Published online: 19 October 2018

\section{References}

1. Burt RW, DiSario JA, Cannon-Albright L. Genetics of colon cancer: impact of inheritance on colon cancer risk. Annu Rev Med. 1995:46:371-9.

2. Petersen GM, Slack J, Nakamura Y. Screening guidelines and premorbid diagnosis of familial adenomatous polyposis using linkage. Gastroenterology. 1991;100:1658-64.

3. Giardiello FM, Krush AJ, Petersen GM, Booker SV, Kerr M, Tong LL, et al. Phenotypic variability of familial adenomatous polyposis in 11 unrelated families with identical APC gene mutation. Gastroenterology. 1994;106: 1542-7.

4. Friedl W, Caspari R, Sengteller M, Uhlhaas S, Lamberti C, Jungck M, et al. Can APC mutation analysis contribute to therapeutic decisions in familial adenomatous polyposis? Experience from 680 FAP families. Gut. 2001;48: 515-21.

5. Jasperson KW, Tuohy TM, Neklason DW, Burt RW. Hereditary and familial Colon Cancer. Gastroenterology. 2010;138:2044-58.

6. Li H. Aligning sequence reads, clone sequences and assembly contigs with BWA-MEM. arXiv:13033997 [q-bio]. 2013. http://arxiv.org/abs/1303.3997. Accessed 4 Sep 2018

7. DePristo MA, Banks E, Poplin R, Garimella KV, Maguire JR, Hartl C, et al. A framework for variation discovery and genotyping using next-generation DNA sequencing data. Nat Genet. 2011;43:491-8.

8. Hamilton W, Round A, Sharp D, Peters TJ. Clinical features of colorecta cancer before diagnosis: a population-based case-control study. $\mathrm{Br} J$ Cancer. 2005:93:399-405.

9. Thompson MR, O'Leary DP, Flashman K, Asiimwe A, Ellis BG, Senapati A. Clinical assessment to determine the risk of bowel cancer using symptoms, age, mass and Iron deficiency anaemia (SAMI). Br J Surg. 2017;104:1393-404.

10. Landrum MJ, Lee JM, Benson M, Brown GR, Chao C, Chitipiralla S, et al. ClinVar: improving access to variant interpretations and supporting evidence. Nucleic Acids Res. 2018;46:D1062-7.

11. Heinen CD. Genotype to phenotype: analyzing the effects of inherited mutations in colorectal cancer families. Mutat Res. 2010;693:32-45.

12. Half E, Bercovich D, Rozen P. Familial adenomatous polyposis. Orphanet J Rare Dis. 2009;4:22.

13. Dihlmann S, Gebert J, Siermann A, Herfarth C, von Knebel Doeberitz M. dominant negative effect of the APC1309 mutation: a possible explanation for genotype-phenotype correlations in familial adenomatous polyposis. Cancer Res. 1999:59(8):1857-60.

14. Miyoshi $Y$, Ando H, Nagase H, Nishisho I, Horii A, Miki Y, et al. Germ-line mutations of the APC gene in 53 familial adenomatous polyposis patients. Proc Natl Acad Sci. 1992;89:4452-6.

15. Cottrell S, Bodmer WF, Bicknell D, Kaklamanis L. Molecular analysis of APC mutations in familial adenomatous polyposis and sporadic colon carcinomas. Lancet. 1992:340:626-30.

16. Varesco L, Gismondi V, James R, Robertson M, Grammatico P, Groden J, et al. Identification of APC gene mutations in Italian adenomatous polyposis coli patients by PCR-SSCP analysis. Am J Hum Genet. 1993;52:280-5.

17. De Benedetti L, Sciallero S, Gismondi V, James R, Bafico A, Biticchi R, et al. Association of APC gene mutations and histological characteristics of colorectal adenomas. Cancer Res. 1994;54:3553-6.

18. Yagi OK, Akiyama Y, Ohkura Y, Ban S, Endo M, Saitoh K, et al. Analyses of the APC and TGF-beta type II receptor genes, and microsatellite instability in mucosal colorectal carcinomas. Jpn J Cancer Res. 1997;88:718-24.

19. Gryfe R, Di Nicola N, Gallinger S, Redston M. Somatic instability of the APC I1307K allele in colorectal neoplasia. Cancer Res. 1998:58:4040-3.

20. Sánchez-de-Abajo A, de la Hoya M, van Puijenbroek M, Tosar A, LópezAsenjo JA, Díaz-Rubio E, et al. Molecular analysis of colorectal cancer tumors from patients with mismatch repair proficient hereditary nonpolyposis colorectal cancer suggests novel carcinogenic pathways. Clin Cancer Res. 2007;13:5729-35.

21. Syed Sameer A, Shah ZA, Abdullah S, Chowdri NA, Siddiqi MA. Analysis of molecular aberrations of Wnt pathway gladiators in colorectal cancer in the Kashmiri population. Hum Genomics. 2011;5:441-52.

22. Christie M, Jorissen RN, Mouradov D, Sakthianandeswaren A, Li S, Day F, et al. Different APC genotypes in proximal and distal sporadic colorectal cancers suggest distinct WNT/B-catenin signalling thresholds for tumourigenesis. Oncogene. 2013;32:4675-82.
23. Chen Q-W, Zhang X-M, Zhou J-N, Zhou X, Ma G-J, Zhu M, et al. Analysis of small fragment deletions of the APC gene in Chinese patients with familial adenomatous polyposis, a precancerous condition. Asian Pac J Cancer Prev. 2015;16:4915-20.

24. Kamps R, Brandão R, Bosch B, Paulussen A, Xanthoulea S, Blok M, et al. Nextgeneration sequencing in oncology: genetic diagnosis, risk prediction and Cancer classification. Int J Mol Sci. 2017:18:308.
Ready to submit your research? Choose BMC and benefit from:

- fast, convenient online submission

- thorough peer review by experienced researchers in your field

- rapid publication on acceptance

- support for research data, including large and complex data types

- gold Open Access which fosters wider collaboration and increased citations

- maximum visibility for your research: over $100 \mathrm{M}$ website views per year

At BMC, research is always in progress.

Learn more biomedcentral.com/submissions 